\title{
Superresolution with Optically-Motivated Blind Deconvolution
}

\author{
Ferréol Soulez, Michael Unser \\ Biomedical Imaging Group, École polytechnique fédérale de Lausanne (EPFL), CH-1015 Lausanne, Switzerland. \\ ferreol.soulez@epfl.ch
}

\begin{abstract}
We show in this paper that an optically motivated parameterization of the PSF prevents classical degeneracies of the blind deconvolution in widefield fluorescence microscopy and provides a resolution close to SIM.
\end{abstract}

OCIS codes: $100.1455,180.2520$

\section{Introduction}

Widefield fluorescence microscopy is the simplest setup in biological imaging. It consists of imaging fluorescent cellular structures under uniform illumination. Moving the focal plane through the sample gives a 3D representation of the object. Unfortunately, this representation suffers from coarse resolution, especially along the optical axis. Image formation is modeled by a $3 \mathrm{D}$ convolution of the $N$-pixel object $\boldsymbol{f} \in \mathbb{R}^{N}$ by the point-spread function (PSF) $\boldsymbol{h} \in \mathbb{R}^{N}$ to yield the direct model of the data $g \in \mathbb{R}^{N}$ :

$$
\boldsymbol{g}=\mathbf{H} \boldsymbol{f}+\boldsymbol{n},
$$

where $\boldsymbol{n} \in \mathbb{R}^{N}$ accounts for some additive noise and $\mathbf{H}$ is a 3D convolution matrix derived from the PSF $h \in L_{2}\left(\mathbb{R}^{3}\right)$ The resolution of the setup is given by the bandwidth of the 3D PSF. To improve the resolution, one can recover the object $f$ from the blurred data $g$ using a deconvolution algorithm (see [1] and references therein). It is important to notice that the PSF $\boldsymbol{h}$ must be provided to these deconvolution algorithms. Unfortunately, the PSF is rarely known and most methods rely on theoretical diffraction-limited PSF or experimental PSF measured using calibration beads. By simultaneously estimating the PSF and the object from the data only, blind deconvolution algorithms bypass the problem of PSF calibration.

Few blind deconvolution algorithms have been proposed for fluorescence microscopy [2-7] because blind deconvolution is a very challenging problem as it is strongly ill-posed and non linear. We present here a PSF model that prevents the classical degeneracies of blind deconvolution ending in a well-posed but still non linear inverse problem. Our model is derived from Fourier optics theory, where the PSF is modeled as the power spectrum of a complex pupil function. Used in a blind deconvolution algorithm [6], this model provides, on real data, a resolution close to that of structured-illumination microscopy (SIM).

\section{The PSF model}

In optical setups, the 2D image in the detector plane of a monochromatic sub-resolved spot in the focal plane (i.e., the PSF $h(x, y, 0))$ is fully described by the complex amplitude of the light in the exit pupil plane, namely, the pupil function $p\left(\omega_{x}, \omega_{y}\right)$. The defocalization of this spot by $z$ only amounts to adding to the phase of the pupil the defocus term $z d\left(\omega_{x}, \omega_{y}\right)=z \sqrt{\left(n_{\mathrm{i}} / \lambda\right)^{2}-\left(\omega_{x}-\kappa_{x}\right)^{2}-\left(\omega_{y}-\kappa_{y}\right)^{2}}$, where $n_{\mathrm{i}}$ is the refractive index of the immersion medium and $\lambda$ is the wavelength. The term $\boldsymbol{\kappa}=\left\{\kappa_{x}, \kappa_{y}\right\}$ accounts for misalignment of the optical axis and the center of the pupil. The 3D PSF is built stacking such 2D PSFs and by varying the amount of defocus. Under a Fresnel approximation, the 2D PSFs are linked to the pupil function by a Fourier transform, as

$$
h(x, y, z)=\left|\iint p\left(\omega_{x}, \omega_{y}\right) \exp \left(2 \mathrm{i} \pi z d\left(\omega_{x}, \omega_{y}\right)\right) \exp \left(2 \mathrm{i} \pi\left(\omega_{x} x+\omega_{y} y\right)\right) \mathrm{d} \omega_{x} \mathrm{~d} \omega_{y}\right|^{2} .
$$




\begin{tabular}{lll}
\hline degeneracies & & effect of PSF parameterization \\
\hline swap & $f * h=h * f$ & object cannot be described as a power-spectrum \\
scale & $(\alpha f) *\left(\frac{1}{\alpha} h\right)=f * h$, & PSF is normalized $\|\boldsymbol{h}\|_{1}=1$ \\
no blur & $\delta * g=g$ & PSF is bandlimited $h \neq \delta$ \\
shift & $f * h=\left(\delta_{-s} * f\right) *\left(\delta_{s} * h\right)$ & PSF is centered if $\alpha_{1}=\alpha_{2}=0$ \\
reducibility & $(g * f) * h=f *(* h)$ & PSF described as the power spectrum of a pupil function is irreducible [9] \\
inversion & $f * h=(s * f) *\left(s^{-1} * h\right)$ & PSF is irreducible \\
\hline
\end{tabular}

Table 1. Effect of the PSF parameterization on blind deconvolution degeneracies

The pupil is non-zero on the disk defined by $\sqrt{\omega_{x}^{2}+\omega_{y}^{2}} \leq \mathrm{NA} / \lambda$. Thus, Zernike polynomials $Z_{n}$ provide a suitable basis to express both the modulus $\rho\left(\omega_{x}, \omega_{y}\right)$ and the phase $\phi\left(\omega_{x}, \omega_{y}\right)$ of the pupil function $p$ as

$$
\begin{aligned}
& p\left(\omega_{x}, \omega_{y}\right)=\rho\left(\omega_{x}, \omega_{y}\right) \exp \left(\iota \phi\left(\omega_{x}, \omega_{y}\right)\right), \\
& \rho\left(\omega_{x}, \omega_{y}\right)=\sum_{n} \beta_{n} Z_{n}\left(\omega_{x}, \omega_{y}\right), \\
& \phi\left(\omega_{x}, \omega_{y}\right)=\sum_{n} \alpha_{n} Z_{n}\left(\omega_{x}, \omega_{y}\right) .
\end{aligned}
$$

As the phase piston has no effect on the resulting PSF, we set $\alpha_{0}=0$. Similarly, we canceling the tip-tilt parameters $\left(\alpha_{1}=\alpha_{2}=0\right)$ to keep the PSF laterally centered. As the PSF is non-negative, PSF normalization (i.e., energy conservation) can be expressed as the $\ell_{1}$ norm constraint $\|\boldsymbol{h}\|_{1}=1$. This constraint can can be written as an $\ell_{2}$ constraint on the modulus expressed in the Zernike orthonormal basis: $\|\boldsymbol{\beta}\|_{2}=1$.

\section{Blind Deconvolution Algorithm}

To solve the blind deconvolution, we have to find the optimal parameters $\left\{\boldsymbol{f}^{+}, \boldsymbol{\alpha}^{+}, \boldsymbol{\beta}^{+}, \boldsymbol{\kappa}^{+}\right\}$that minimize the cost function $\mathscr{J}(\boldsymbol{f}, h(\boldsymbol{\alpha}, \boldsymbol{\beta}, \boldsymbol{\kappa}))=\mathscr{L}(\boldsymbol{f}, h(\boldsymbol{\alpha}, \boldsymbol{\beta}, \boldsymbol{\kappa}))+\mathscr{R}(\boldsymbol{f})$ with $\boldsymbol{f} \geq 0$. This function is a sum of the data term $\mathscr{L}(\boldsymbol{f}, h(\boldsymbol{\alpha}, \boldsymbol{\beta}, \boldsymbol{\kappa}))$ and the regularization functional $\mathscr{R}(\boldsymbol{f})$. As described in [6], the data term is given by the negloglikelihood of a Gaussian non-stationary noise. The regularization is the classical 3D total variation.

Due to the non linearity of this cost function and because these parameters have different physical meanings, the simultaneous estimation of both the object $\boldsymbol{x}$ and the PSF $h(\boldsymbol{\alpha}, \boldsymbol{\beta}, \boldsymbol{\kappa})$ is known to be very badly conditioned. It thus relies on an alternating-minimization scheme [8]. Each outer iteration of this alternating minimization consists of estimating the object while keeping the PSF fixed and then using this estimated object to estimate successively PSF parameters: defocus $\boldsymbol{\kappa}$; phase $\boldsymbol{\alpha}$; and modulus $\boldsymbol{\beta}$.

\section{Blind Deconvolution Degeneracies}

The blind deconvolution problem is ill-posed, with twice as many unknowns as measurements. Most notably, there exist infinitely many couples $\{f, h\}$ such that $f * h=g$. We believe that our optically motivated model prevents all these degeneracies as described in Table 1.

\section{Results}

We illustrate the effectiveness of our blind deconvolution method by processing experimental data shared by Cazares et al. on the cell image library ${ }^{1}$. It is a micrograph of microtubules in a Drosophila S2 cell labeled by Alexa Fluor 488 and observed with a Zeiss Elyra SIM with a NA $=1.4$ objective. It is composed of a 3D widefield image and a superresolution SIM image that can be used as ground truth. Both micrographs have $(1024 \times 1024 \times 44)$ voxels, each voxel being of size $40 \times 40 \times 110 \mathrm{~nm}^{3}$.

The data, the blind deconvolution result, and the structured-illumination image are presented in Figure 1. Compared to widefield, the blind deconvolution result shows an increase in resolution, especially along the axial dimension. This is clearly visible on the cuts across a microtubule presented in Figure 5. As microtubule are under-resolved by the microscope, their estimated width provides a good proxy of the resolution. On the presented cuts, the resolution after blind deconvolution is not much coarser than that of the SIM for a much less complicated setup.

\footnotetext{
${ }^{1}$ http://www.cellimagelibrary.org/images/36797
} 


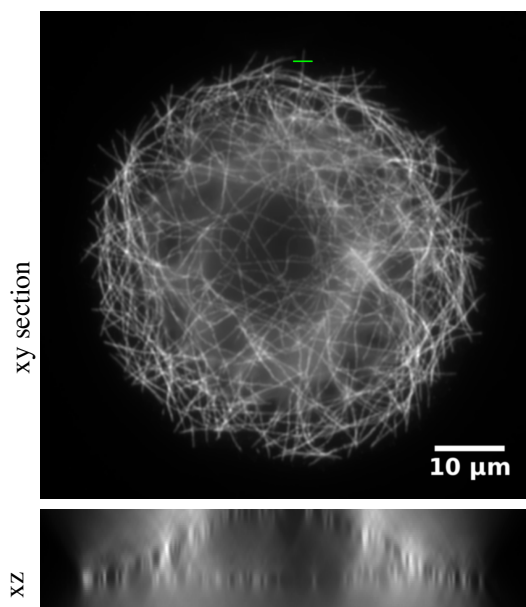

(a) data
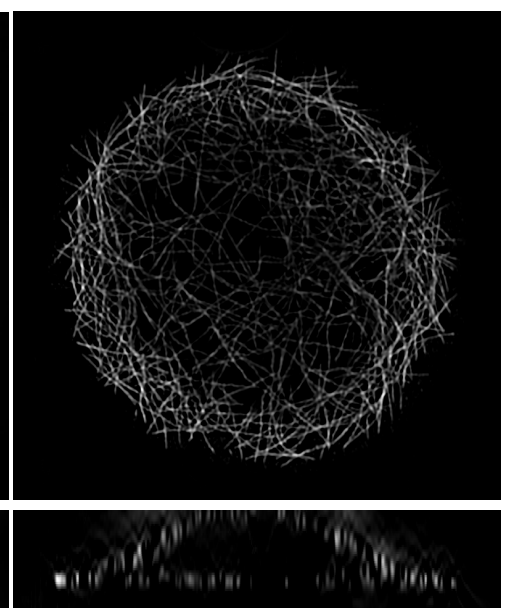

(b) Blind deconvolution

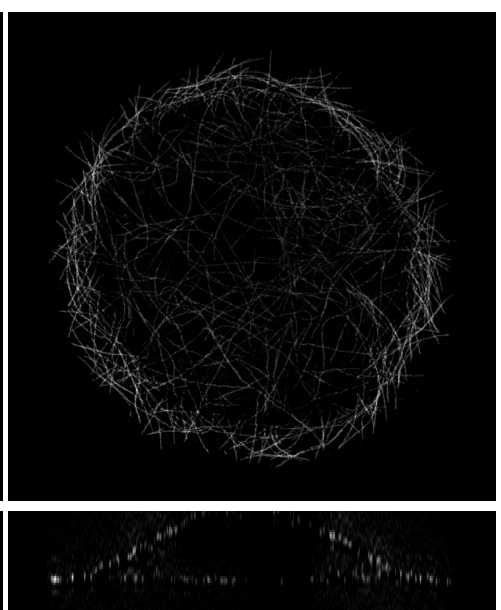

(c) SIM

Fig. 1. Widefield observation and its blind deconvolution compared to the superresolution image given by structured illumination (SIM) on a drosophila S2 cell.

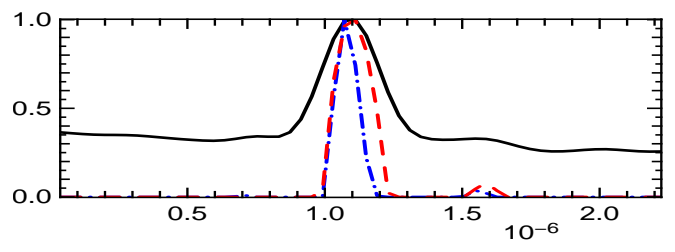

(a) lateral (xy) section

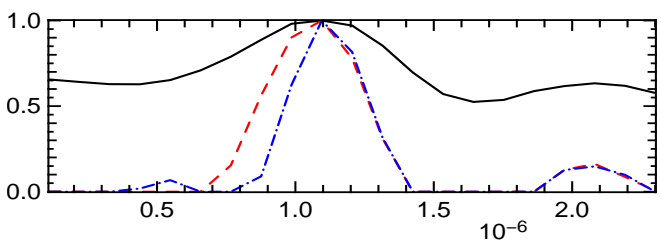

(b) axial (xz) section

Fig. 2. Lateral and axial profile of the microtubule indicated by the green line on Figure 1.a for the wide field data (solid line), its blind deconvolution (dashed red), and the SIM (mixed blue).

\section{Acknowledgments}

This work is supported by the Sinergia project "Euclid" from the Swiss National Science Foundation.

\section{References}

1. P. Sarder and A. Nehorai, "Deconvolution methods for 3-d fluorescence microscopy images," Signal Processing Magazine, IEEE 23, 32-45 (2006).

2. J. Markham and J.-A. Conchello, "Parametric blind deconvolution: a robust method for the simultaneous estimation of image and blur," J. Opt. Soc. Am. A 16, 2377-2391 (1999).

3. E. F. Y. Hom et al., "Aida: an adaptive image deconvolution algorithm with application to multi-frame and three-dimensional data,” J. Opt. Soc. Am. A 24, 1580-1600 (2007).

4. L. M. Mugnier et al., "Mistral: a myopic edge-preserving image restoration method, with application to astronomical adaptive-optics-corrected long-exposure images," J. Opt. Soc. Am. A 21, 1841-1854 (2004).

5. T. Kenig et al., "Blind image deconvolution using machine learning for three-dimensional microscopy," Pattern Analysis and Machine Intelligence, IEEE Transactions on 32, 2191-2204 (2010).

6. F. Soulez et al., "Blind deconvolution of 3d data in wide field fluorescence microscopy," in "proceedings of the 9th International Symposium on Biological Imaging," (Barcelona, 2012).

7. M. Keuper et al., "Blind deconvolution of widefield fluorescence microscopic data by regularization of the optical transfer function (otf)," in "IEEE CVPR" (2013).

8. Y. You and M. Kaveh, "A regularization approach to joint blur identification and image restoration," Image Processing, IEEE Transactions on 5, 416-428 (1996).

9. A. Fannjiang, “Absolute uniqueness of phase retrieval with random illumination,” Inverse Problems 28, 075,008 (2012). 